Hausmittel im Test

\title{
Mit Krautwickel gegen Knieschmerzen
}

— Seit Jahrhunderten wird Weißkraut in der naturheilkundlichen Medizin eingesetzt, etwa als Brustwickel bei stillenden Frauen mit Milchstau. Auch arthrosebedingte Gelenkschmerzen soll ein Wickel aus Weißkrautblättern lindern. Romy Lauche von der Klinik für Naturheilkunde und Integrative Medizin in Essen und ihre Kollegen haben die Wirkung des Kohlwickels bei Patienten mit Kniegelenksarthrose Grad II - III (Kellgren-Lawrence-Score) untersucht [Clin J Pain 2016; online 17. Februar].

An der Studie nahmen 81 Patienten teil, die im Mittel 65 Jahre alt waren. Die Patienten der ersten Gruppe (CWL-Gruppe) wurden angehalten, vier Wochen lang einmal täglich für mindestens zwei Stunden oder besser über Nacht am schmerzenden Knie einen Krautwickel aufzulegen. Die Probanden der zweiten Gruppe (TPG-Gruppe) sollten einmal täglich das Knie mit $1-4 \mathrm{~g}$ Diclofenac-haltigem Schmerzgel einreiben und die der dritten Gruppe (UC-Gruppe) die
Behandlung weiterführen, die sie bereits vor der Studie angewandt hatten.

Hatten die Probanden der drei Gruppen zu Beginn der Studien die Schmerzintensität ähnlich eingeschätzt, gab die Patientengruppe, die Krautwickel aufgelegt hatte, nach vier Wochen weniger Schmerzen an als die UC-Gruppe. Zwischen CWL- und TPGGruppe hingegen fand sich kein Unterschied $(-8,6 ; 95 \%-\mathrm{KI}-21,5$ bis 4,$4 ; \mathrm{p}=0,190)$.

Nach zwölf Wochen waren alle drei Gruppen in puncto Schmerzintensität wieder gleich auf. Bei der Gelenkfunktionalität (WOMACScore, $30 \mathrm{sec}$ CST) und Lebensqualität (SF36) ergab sich für die Krautwickelbehandlung im Vergleich zur gewohnten Therapie ein klarer Vorteil.

Verglichen mit der Schmerzgelgruppe beurteilten die Krautwickelpatienten nach vier Wochen die Funktionalität des Kniegelenkes etwas besser (WOMAC-Index) und nach zwölf Wochen die allgemeine Lebensqualität (SF-36).

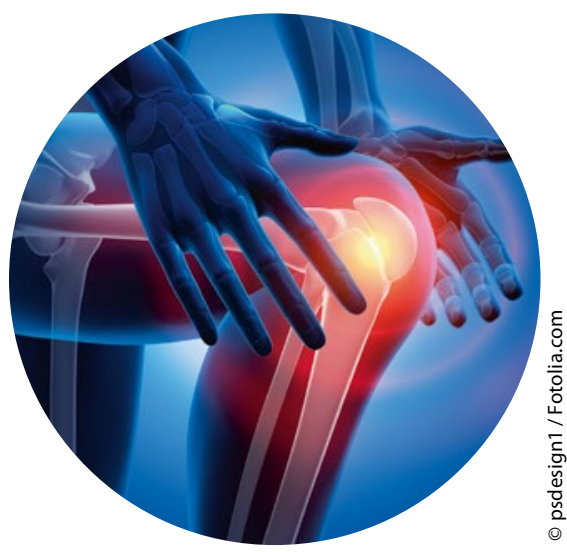

Nach einer vierwöchigen Behandlungsdauer schnitt der Krautwickel in puncto Schmerz, Gelenkfunktion und Lebensqualität besser ab als die "vorherige" Therapie. Im Vergleich zur Schmerzgelapplikation zeigte sich aber kein Vorteil, fassen die Autoren zusammen. Ihrer Meinung nach könne diese naturheilkundliche Behandlungsmethode bei Gelenkarthrose guten Gewissens empfohlen werden, aufgrund des guten Sicherheitsprofils auch zur langfristigen Anwendung. Weitere Studien seien aber wünschenswert.

\section{Barmer-Arztreport}

\section{Schmerzpatienten unterversorgt}

_ In Deutschland leiden etwa 3,25 Millionen Menschen unter chronischen Schmerzen. Ihre Zahl hat sich von 2005 bis 2014 mehr als verdoppelt. Das geht aus dem aktuellen Arztreport der Barmer GEK hervor.

"Die Bekämpfung des chronischen Schmerzes muss zu einem nationalen Gesundheitsziel erklärt werden", fordert Dr. Christoph Straub, Vorstandsvorsitzender der Barmer GEK. Hintergrund ist der aktuelle Arztreport seiner Kasse. Demnach litten 2014 etwa 4\% der Deutschen unter chronischen Schmerzen. Das AQUA-Institut aus Göttingen hatte für den Report die Daten der acht Millionen Barmer-Versicherten ausgewertet und auf die Bevölkerung hochgerechnet. „Wir brauchen nicht weitere Angebote, sondern eine gut vernetzte und interdisziplinäre Versorgung", so Straub und Studienleiter Professor Joachim Szecsenyi.
Handlungsbedarf sieht Straub sowohl im ambulanten als auch im stationären Bereich: „Wir brauchen engagierte Hausärzte und eine effiziente Behandlung in Kliniken." Die Zahl der niedergelassenen Ärzte, die über eine schmerztherapeutische Zusatzqualifikation verfügen, ist von 2009 bis 2014 um $15 \%$ auf 1.142 Ärzte gestiegen. Insgesamt wurden 655.000 Patienten im Jahr 2014 ambulant versorgt, $27 \%$ mehr als 2009.

Die Kapazitäten im stationären Sektor sind deutlich stärker gestiegen. Laut Arztreport hat sich die Zahl der Kliniken verdoppelt, die eine multimodale Schmerztherapie anbieten. 61.000 Patienten waren 2014 stationär versorgt worden. „Das ist jedoch nur ein Fünftel aller Patienten, die für eine solche Therapie infrage kommen", meint Straub. Die Krankenhäuser setzen zunehmend auf kurze Verweildauern. "Damit sinkt die Effektivität",

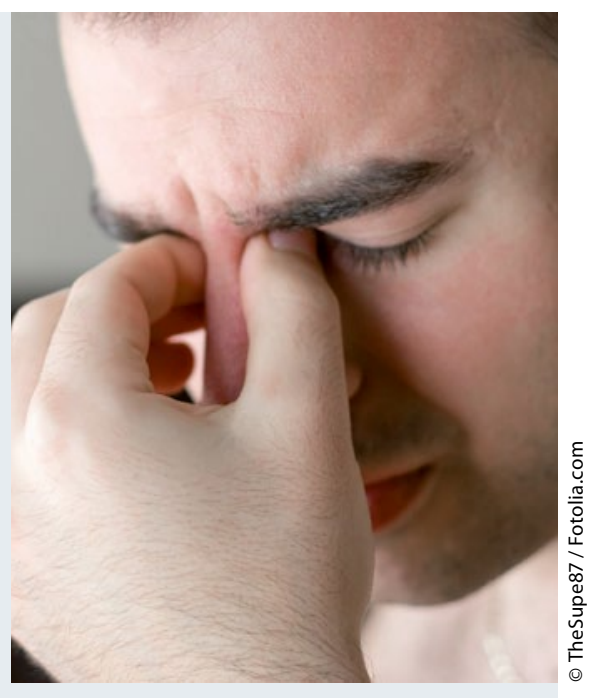

betont Straub. Um die Betroffenen gut zu behandeln, so Straub weiter, sei eine "durchgängige Versorgungskette nötig, in der interdisziplinär zusammen gearbeitet wird“. Der Hausarzt sollte dabei eine Lotsenfunktion übernehmen.

Susanne Werner 